\title{
Electronic structure of the electron-doped $\mathrm{Ca}_{0.86} \operatorname{Pr}_{0.14} \mathrm{MnO}_{3}$
}

\author{
M. K. Dalai, ${ }^{1,2}$ P. Pal, ${ }^{1,2}$ B. R. Sekhar, ${ }^{1}$ M. Merz, ${ }^{3}$ P. Nagel, ${ }^{3}$ S. Schuppler, ${ }^{3}$ and C. Martin ${ }^{4}$ \\ ${ }^{1}$ Institute of Physics, Sachivalaya Marg, Bhubaneswar 751005, India \\ ${ }^{2}$ National Physical Laboratory, Dr. K. S. Krishanan Marg, New Delhi 110012, India \\ ${ }^{3}$ Karlsruhe Institute of Technology, Institut fuer Festkoerperphysik, 76021 Karlsruhe, Germany \\ ${ }^{4}$ Laboratoire CRISMAT, UMR 6508, ISMRA, Boulevard du Marechal Juin, 14050, Caen, France \\ (Received 1 March 2011; revised manuscript received 7 February 2012; published 17 April 2012)
}

\begin{abstract}
We have studied the temperature-dependent changes in the near $E_{F}$ electronic structure of the electron-doped, phase separated colossal magnetoresistance (CMR) compound, $\mathrm{Ca}_{0.86} \mathrm{Pr}_{0.14} \mathrm{MnO}_{3}$ using photoemission and x-ray absorption studies. At low temperatures, this compound shows a high $e_{g}$ electron density near the $E_{F}$ though the compound is insulating. Photoemission measurements further showed a temperature dependence of the $e_{g}$ electron occupation and localization while the complementary results of XAS showed corresponding changes in the number of unoccupied states. Our results indicate a transfer of charges from the $t_{2 g}$ states to the $e_{g}$ band, probably due to a decrease in the crystal field splitting in the ferromagnetic metallic (FMM) phase following the structural changes of the $\mathrm{MnO}_{6}$ octahedra at low temperatures. We have interpreted our results from a FMM-antiferromagnetic insulating (AFMI) phase separation scenario.
\end{abstract}

DOI: 10.1103/PhysRevB.85.155128

PACS number(s): 79.60.-i, 75.47.Gk, 71.20.-b

\section{INTRODUCTION}

Strong interplay of charge, spin, and lattice degrees of freedom of electrons form the basis of the phenomena of colossal magnetoresistance (CMR) shown by manganites. To a large extent, the traditional models ${ }^{1-3}$ employing the charge-spin coupling, have been able to explain the CMR in many of the hole-doped compositions $\left(R_{1-x} A_{x} \mathrm{MnO}_{3}\right.$ with $x \ll 1 / 2$, where $R$ is a trivalent lanthanide and $A$ is a divalent alkaline-earth element). But, much less is known about the electron-doped versions of these materials with $x \rightarrow 1$. The charge, orbital and spin ordering add complexity to the ferromagnetic double exchange and the superexchange interactions in these materials. These $\mathrm{Mn}(\mathrm{IV})$ rich compositions exhibit marked differences from their $\mathrm{Mn}$ (III) rich counterparts in their electronic and magnetic properties. For example, their electrical conductivity behavior in the range of 150-300 K was found to be distinctly different from the hole-doped materials. ${ }^{4}$ Further, earlier studies ${ }^{5-7}$ have shown the existence of dynamic ferromagnetic spin correlations at high temperatures and antiferromagnetic correlations at low temperatures in these electron-doped systems. Recent studies $^{8,9}$ on thin film and nanoparticle forms of different CMR compositions have highlighted the role of dimensionality in such phase coexistence. The coexisting magnetic phases in these materials are the focus of new models proposed to explain the metal-insulator transitions in doped $3 d$ metal oxide compounds. ${ }^{10-13}$

It has been shown that substitution of $\mathrm{Ca}$ by a trivalent cation in the G-type antiferromagnet, $\mathrm{CaMnO}_{3}$ leads to the formation of a FM component with a maximum for optimal electron doping. ${ }^{6,14,15}$ Depending on the nature of the trivalent cation, the $x_{\text {opt }}$ was found ${ }^{4,7,15}$ to lie between 0.135 and 0.16 . With low filling of the narrow $e_{g}$ band, these materials have strong electron-electron interactions leading the localization of $e_{g}$ electrons. A. Maignan et al. have shown that such localization could lead to the formation of FM domains (electron rich) embedded in an AFM background (electron poor). ${ }^{6}$ In such phase separated materials, electrical transport is by current percolation through the FM clusters. ${ }^{10,16-19}$ Doping $\operatorname{Pr}$ in $\mathrm{Ca}_{1-x} \mathrm{Pr}_{x} \mathrm{MnO}_{3}$ was found to result in a ferromagnetic metallic (FMM) state for $x \leqslant 0.13$ and an antiferromagnetic insulating state (AFMI) for $x \geqslant 0.14$ at low temperatures. ${ }^{4,7,20}$ It has been shown that this FMM state is qualitatively different from the FMM state observed in hole-doped CMR systems. ${ }^{6}$ We have chosen the $\mathrm{Ca}_{0.86} \operatorname{Pr}_{0.14} \mathrm{MnO}_{3}$ composition, which lies at the boundary of the compositions with two different magnetic ground states. Here, we have studied the near-Fermi-level $\left(E_{F}\right)$ occupied and unoccupied electronic structure of the $\mathrm{Ca}_{0.86} \mathrm{Pr}_{0.14} \mathrm{MnO}_{3}$ using photoemission (PES) and $\mathrm{O}$ K-edge $\mathrm{x}$-ray absorption spectroscopy (XAS).

\section{EXPERIMENT}

The polycrystalline sample of $\mathrm{Ca}_{0.86} \mathrm{Pr}_{0.14} \mathrm{MnO}_{3}$ was prepared by standard solid state reactions. Details of the sample preparation technique are published elsewhere. ${ }^{4}$ Purity, homogeneity, and composition of the sample were confirmed by using electron diffraction and energy dispersive spectroscopy. The electrical and magnetic properties of this composition have also been published earlier. ${ }^{4,20}$ This sample shows a metallic behavior down to $\sim 200 \mathrm{~K}$ and followed by a slight increase till $110 \mathrm{~K}$ (see Fig. 1). Below the $110 \mathrm{~K}$, the resistivity goes up by many orders of magnitude. The conductivity behavior of this sample is, in general, similar to that of other electron-doped CMR systems. ${ }^{6,7,14,21}$

Photoemission and $\mathrm{x}$-ray absorption spectroscopy measurements were performed by using the light from IFP's soft $\mathrm{x}$-ray beamline WERA at the ANKA synchrotron light source, Karlsruhe, Germany. The PES measurements were carried out using the photon energy $124 \mathrm{eV}$. The photoelectrons were collected using a Scienta SES 2002 electron energy analyzer. The energy resolution was $25 \mathrm{meV}$ in case of the narrow scans $(-2-2.5 \mathrm{eV})$ and it was $125 \mathrm{meV}$ in case of wide scans $(-2-15 \mathrm{eV})$ of the valence band. The O K-edge XAS spectra has been taken in the fluorescence detection mode and the energy resolution was set to $150 \mathrm{meV}$. The sample surface was 


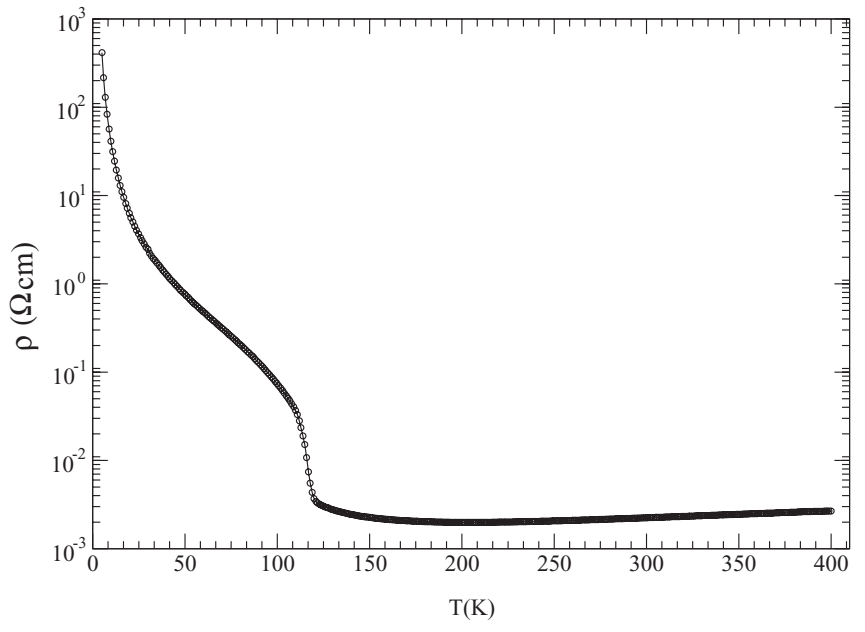

FIG. 1. The resistivity vs temperature curve of $\mathrm{Ca}_{0.86} \mathrm{Pr}_{0.14} \mathrm{MnO}_{3}$.

cut using a diamond knife under a base vacuum of $\sim 1.0 \times$ $10^{-10}$ mbar before taking the data for each temperature. In case of the photoemission measurements, scraping was repeated until negligible intensity was found for the bump around 9-10 eV, which is a signature of surface contamination. ${ }^{22}$ For the temperature-dependent measurements, a liquid-helium cryostat was used, with a $\mathrm{Si}$ diode for monitoring the temperature.

\section{RESULTS AND DISCUSSION}

Figure 2 shows the photoemission spectra of the $\mathrm{Ca}_{0.86} \operatorname{Pr}_{0.14} \mathrm{MnO}_{3}$. Various spectra, taken at 30, 70, 110, 220, and $300 \mathrm{~K}$ show the temperature-dependent changes in the valence-band electronic structure. Intensities of all the spectra were normalized and shifted along the ordinate axis by a constant value for the clarity of presentation. All the features seen in the spectra are dominated by the states due to Mn $3 d-\mathrm{O} 2 p$ hybridization. The broad feature appearing at $\sim 6 \mathrm{eV}$

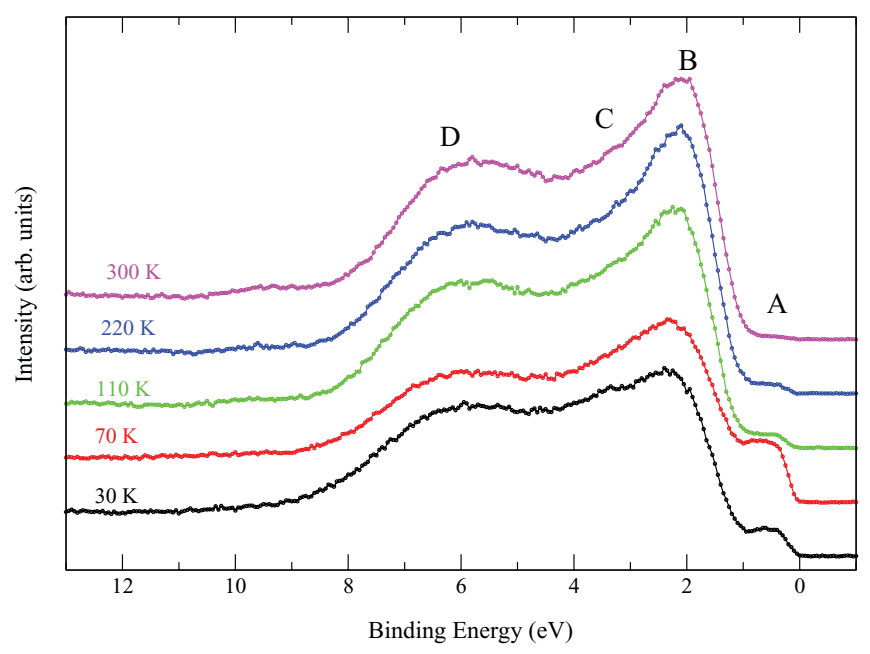

FIG. 2. (Color online) The angle-integrated valence-band photoemission spectrum of $\mathrm{Ca}_{0.86} \mathrm{Pr}_{0.14} \mathrm{MnO}_{3}$ taken by using $124 \mathrm{eV}$ photons. The spectra taken at different temperatures $(30,70,110$, 220 , and $300 \mathrm{~K}$ ) are normalized for their intensities and shifted along $y$ axis by a constant for clarity of presentation.

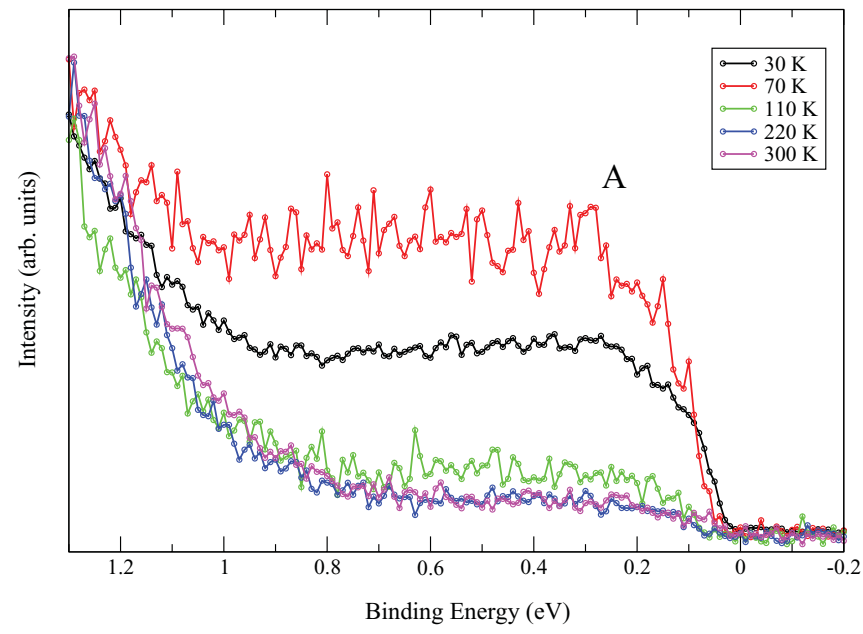

FIG. 3. (Color online) The near $E_{F}$ region of the valence band spectra (with higher resolution) of $\mathrm{Ca}_{0.86} \mathrm{Pr}_{0.14} \mathrm{MnO}_{3}$ taken at temperatures $30,70,110,220$, and $300 \mathrm{~K}$. This feature refers to the Mn $3 d e_{g \uparrow}$ occupied states.

(marked D) is due to the bonding states of this hybridization, while its nonbonding states appear as a shoulder at $\sim 3.2 \mathrm{eV}$ (marked C). The peak at $\sim 2 \mathrm{eV}$ (marked B) is due to the Mn $3 d t_{2 g \uparrow}$ states of the $\mathrm{MnO}_{6}$ octahedra. The Mn $3 d e_{g \uparrow}$ states appear around $\sim 0.3 \mathrm{eV}$ (marked A) in the spectra. A discussion on these spectral features can be found in earlier reports. $^{23-28}$ It should be noted from the figure that with decrease in temperature the intensity of the feature A increases, while that of the features $\mathrm{B}$ and $\mathrm{C}$ decrease. Temperature dependence of the Mn $3 d e_{g \uparrow}$ states is more clearly visible in Fig. 3 where the spectra are taken with a higher resolution. The spectra taken near $300 \mathrm{~K}$ show low, but finite density of states near the $E_{F}$, though the sample shows a metallic behavior near this temperature (see Fig. 1). This is typical of these perovskite transition metal oxide systems that are bad metals and have low charge carrier density. ${ }^{23-25}$ Below 100 $\mathrm{K}$, the intensity of feature A shows a substantial enhancement $(70 \mathrm{~K})$ and then a decrease $(30 \mathrm{~K})$ with temperature. The density of these Mn $3 d e_{g \uparrow}$ states is important to the physical properties, especially the electrical and magnetic behavior of CMR systems. The observed changes in these states will be discussed in the following paragraphs.

In order to see the corresponding changes in the unoccupied states, we have studied our sample using $\mathrm{X}$-ray absorption spectroscopy (XAS). In Fig. 4, we present the O K-edge $\mathrm{X}$-ray absorption spectra (XAS) of the $\mathrm{Ca}_{0.86} \mathrm{Pr}_{0.14} \mathrm{MnO}_{3}$ sample taken at different temperatures. Here, all the spectra have been normalized to the incoming intensity and absolute cross sections and are also corrected for self-absorption and saturation effects (SAC). The method used for SAC was outlined elsewhere. ${ }^{29}$ The spectra show three broad features. The one between 527 to $533 \mathrm{eV}$, called the pre-edge feature in the $\mathrm{O} \mathrm{K}$-edge spectra, is mostly due to the strongly hybridized Mn $3 d-\mathrm{O} 2 p$ orbitals. The feature around $536.5 \mathrm{eV}$ is due to the bands from hybridized $\mathrm{Pr} 5 d$ and $\mathrm{Ca} 3 d$ orbitals, while the structure above $540 \mathrm{eV}$ originates from the states like Mn $4 s p$ and Pr $6 s p$, etc. These assignments of the features are consistent with the earlier reported experiments and band 


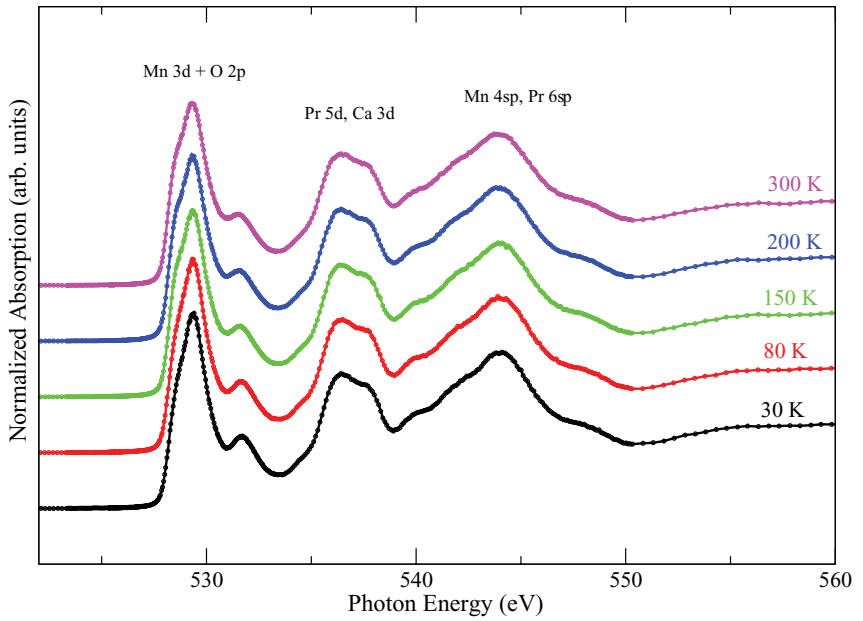

FIG. 4. (Color online) The O K-edge $\mathrm{x}$-ray absorption spectra of the $\mathrm{Ca}_{0.86} \operatorname{Pr}_{0.14} \mathrm{MnO}_{3}$ taken at $30,80,150,200$, and $300 \mathrm{~K}$. The pre-edge feature appears between $527-533 \mathrm{eV}$ is mostly due to the strong hybridization between $\mathrm{Mn} 3 d$ and $\mathrm{O} 2 p$ orbitals.

structure calculations on similar systems. ${ }^{25,30,31}$ The pre-edge region of the $\mathrm{O} \mathrm{K}$-edge XAS has earlier been shown to represent the near $E_{F}$ unoccupied states in these kind of compounds. $^{25,30,31}$ Figure 5 shows the pre-edge region of the XAS spectra of our sample taken at different temperatures. The feature extending from $\sim 527$ to $531 \mathrm{eV}$ consists of two lines. The first one is due to the $\mathrm{O} 2 p$ orbitals hybridized with the Mn $3 d e_{g \uparrow}$ (marked $\mathrm{A}^{\prime}$ ), while the second arises from the $\mathrm{O} 2 p$-Mn $3 d t_{2 g \downarrow}$ hybridization (marked $\mathrm{B}^{\prime}$ ). The weak feature centered around $\sim 531.5 \mathrm{eV}$ corresponds to the $e_{g \downarrow}$ (marked $\mathrm{C}^{\prime}$ ) states. $^{25,32-35}$ All the features show some temperature dependence. As the temperature decreases from 300 to $30 \mathrm{~K}$, the intensity of $\mathrm{A}^{\prime}$ decreases while that of $\mathrm{B}^{\prime}$ increases. Moreover, both $\mathrm{B}^{\prime}$ and $\mathrm{C}^{\prime}$ shift to higher energy as the temperature is lowered.

The spectral changes observed in the photoemission spectra and the x-ray absorption spectra are complementary to each other. With decrease in temperature, the photoemission spectra

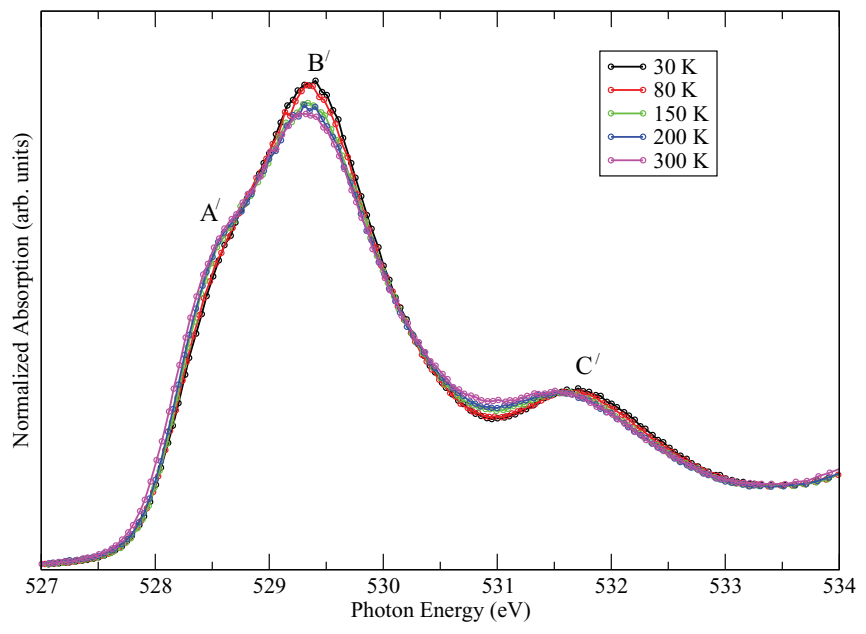

FIG. 5. (Color online) The pre-edge peak of the O K-edge XAS spectra of $\mathrm{Ca}_{0.86} \mathrm{Pr}_{0.14} \mathrm{MnO}_{3}$ taken at temperatures 30, 80, 150, 200, and $300 \mathrm{~K}$. show an increase in the number of Mn $3 d e_{g \uparrow}$ occupied states (A), while the $\mathrm{X}$-ray absorption spectra show a decrease in the Mn $3 d e_{g \uparrow}$ unoccupied states $\left(\mathrm{A}^{\prime}\right)$. Further, an enhancement of this trend below $110 \mathrm{~K}$ is also visible in both photoemission and X-ray absorption spectra. Features $\mathrm{B}$ and $\mathrm{B}^{\prime}$, which originate from the Mn $3 d t_{2 g \uparrow}-\mathrm{O} 2 p$ and $\mathrm{Mn} 3 d t_{2 g \downarrow}-\mathrm{O} 2 p$ hybridized states, respectively, show a concomitant change; intensity of $\mathrm{B}$ decreases and $\mathrm{B}^{\prime}$ increases with decrease in temperature. As mentioned before, the transport, magnetic, and structural properties of the $\mathrm{Ca}_{1-x} \mathrm{Pr}_{x} \mathrm{MnO}_{3}$ system have been reported earlier. ${ }^{4,7,20}$ The parent compound $\mathrm{CaMnO}_{3}$ has an empty $e_{g}$ band with no Jahn-Teller splitting. The metallic behavior shown by the $\mathrm{Ca}_{0.86} \operatorname{Pr}_{0.14} \mathrm{MnO}_{3}$ in the range of $300-200 \mathrm{~K}$ could be understood in terms of the weak Jahn-Teller distortion and thereby weakened electron-phonon coupling. Martin et al. ${ }^{7,20}$ have further shown from neutron diffraction studies that compositions very close to the $\mathrm{Ca}_{0.86} \mathrm{Pr}_{0.14} \mathrm{MnO}_{3}$ consist of FMM clusters embedded in a G-type AFMI matrix. Our spectroscopic results could be understood using this picture of coexisting FMM and AFMI phases. At room temperature, the $e_{g}$ electrons are largely itinerant due to the weak electron-phonon coupling, particularly through the interconnected FMM regions. The metallic behavior shown (see Fig. 1) by the sample in the $300-200 \mathrm{~K}$ range is due to these itinerant charge carriers. As the temperature is lowered from $200 \mathrm{~K}$, these FMM regions become smaller and smaller in size leading to a reduction in the $e_{g}$ band width and a consequent localization of the $e_{g}$ electrons in this band. The spin interaction of these localized electrons further stabilizes the FMM clusters. Below $110 \mathrm{~K}$, the sample turns insulating as the AFM interaction is dominant and the sample now comprises of isolated FMM clusters embedded in an AFMI insulating matrix. The increase in the intensity of $\mathrm{A}$ in the photoemission spectra (see Fig. 2) shows that the $e_{g}$ electron occupation increases with cooling down from 110 to $70 \mathrm{~K}$. Also, since the sample is insulating at these temperatures these $e_{g}$ electrons must be localized in the $e_{g}$ band of the FMM clusters. This increase in the $e_{g}$ electron density and their localization could be due to the weak electron phonon coupling and a possible reduction in the crystal field splitting in FMM phase leading to the transfer of some of the electrons from the $t_{2 g}$ to the $e_{g}$ levels. Correspondingly, the intensity of feature $\mathrm{B}$, which represents the $t_{2 g}$ states, decreases. As expected, on the unoccupied side, the feature $\mathrm{A}^{\prime}$ of the XAS spectra shows a consequent decrease in intensity. Though, as we go from 110 to $70 \mathrm{~K}$, this transfer of electrons and localization in the $e_{g}$ band in the FMM clusters become more pronounced, the spectra collected at $30 \mathrm{~K}$ shows that this process weakens with further cooling. Interestingly, one can see a corresponding change in the slope of the resistivity curve (see Fig. 1). This reduction in the transfer of electrons to the $e_{g}$ band could be due to the shrinking of the FMM regions at these low temperatures and the prevalence of the AFMI phase. Further, the electron localization could be leading to the formation of an insulating gap at the $E_{F}$ below $110 \mathrm{~K}$. Anyway, it should be to note that in this phase separated compound, though the $e_{g}$ electron density near $E_{F}$ is high, the material is an insulator. The strong FMM-AFMI phase separation at low temperatures also seems to modify the $\mathrm{Mn}-\mathrm{O}$ hybridization in this compound. The observed decrease 
in the intensity of $\mathrm{B}$ in the photoemission spectra and the increase in the intensity and shift to higher energies shown by $\mathrm{B}^{\prime}$ and $\mathrm{C}^{\prime}$ in the $\mathrm{X}$-ray absorption spectra indicate a weaker Md $3 d-\mathrm{O} 2 p$ hybridization at low temperatures. These spectral changes are also signatures of a weaker crystal field splitting mentioned before, and a stronger on-site exchange interaction splitting in the AFMI phase. Structural studies reported $^{36,37}$ by Pollert et al. and Jirak et al. have shown that with lowering of temperature the $\mathrm{Ca}_{0.86} \operatorname{Pr}_{0.14} \mathrm{MnO}_{3}$ compound shows a structural change from orthorhombic to an elongated pseudotetragonal structure, where the $\mathrm{MnO}_{6}$ octahedra are elongated along the $z$ direction with four short $a$ - $b$-plane and two long out-of-plane Mn-O-Mn bonds. Results of our study could be of relevance in understanding the differences in nature of such intrinsic phase separations and the extrinsic ones observed in thin films and nanomaterials. ${ }^{8,9}$

\section{CONCLUSIONS}

Using photoemission spectroscopy and O K-edge XAS, we have studied the temperature-dependent changes in the near$E_{F}$ electronic structure of the electron-doped phase-separated CMR compound, $\mathrm{Ca}_{0.86} \operatorname{Pr}_{0.14} \mathrm{MnO}_{3}$. The low-temperature electronic behavior shows marked differences from those of the hole-doped CMR systems, like high $e_{g}$ electron density near $E_{F}$ though the compound is insulating. Results from the photoemission and XAS measurements are complementary to each other. The photoemission showed an increase in the $e_{g}$ electron occupation and localization with decrease in temperature, while the XAS showed a corresponding decrease in the number of unoccupied states. We have interpreted our results from the point of view of a FMM-AFMI phase separation. We also see a transfer of electrons from the $t_{2 g}$ states to the $e_{g}$ band in our photoemission and XAS results, which could possibly be due to a reduced crystal field splitting in the FMM phase at low temperatures. Further, we see temperature-dependent changes in the Mn $3 d-\mathrm{O} 2 p$ hybridization strengths in this compound.

\section{ACKNOWLEDGMENTS}

The authors acknowledge Department of Science and Technology (DST), Government of India for the financial support to carry out the experiment at the WERA beam line of ANKA synchrotron light source, Karlsruhe, Germany. The ANKA synchrotron light source is gratefully acknowledged for the provision of beamtime.
${ }^{1}$ C. Zener, Phys. Rev. 82, 403 (1951).

${ }^{2}$ P. W. Anderson and H. Hasegawa, Phys. Rev. 100, 675 (1955).

${ }^{3}$ P.-G. de Gennes, Phys. Rev. 118, 141 (1960).

${ }^{4}$ A. Maignan, C. Martin, F. Damay, and B. Raveau, Chem. Mater 10, 950 (1998).

${ }^{5}$ W. Bao, J. D. Axe, C. H. Chen, and S. W. Cheong, Phys. Rev. Lett. 78, 543 (1997).

${ }^{6}$ A. Maignan, C. Martin, F. Damay, B. Raveau, and J. Hejtmanek, Phys. Rev. B 58, 2758 (1998).

${ }^{7}$ C. Martin, A. Maignan, M. Hervieu, B. Raveau, Z. Jirak, M. M. Savosta, A. Kurbakov, V. Trounov, G. Andre, and F. Bouree, Phys. Rev. B 62, 6442 (2000)

${ }^{8}$ C. Aruta, G. Ghiringhelli, V. Bisogni, L. Braicovich, N. B. Brookes, A. Tebano, and G. Balestrino, Phys. Rev. B 80, 014431 (2009).

${ }^{9}$ Yang Wang and Hong Jin Fan, Phys. Rev. B 83, 224409 (2011).

${ }^{10}$ Shun-Qing Shen and Z. D. Wang, Phys. Rev. B 58, 8877R (1998).

${ }^{11}$ A. J. Millis, P. B. Littlewood, and B. I. Shraiman, Phys. Rev. Lett. 74, 5144 (1995).

${ }^{12}$ A. J. Millis, B. I. Shraiman, and R. Mueller, Phys. Rev. Lett. 77, 175 (1996).

${ }^{13}$ A. J. Millis, Nature (London) 392, 147 (1998).

${ }^{14}$ M.M. Savosta, P. Novak, M. Marysko, Z. Jirk, J. Hejtmanek, J. Englich, J. Kohout, C. Martin, and B. Raveau, Phys. Rev. B 62, 9532 (2000)

${ }^{15}$ J. J. Neumeier and J. L. Cohn, Phys. Rev. B 61, 14319 (2000).

${ }^{16}$ A. Moreo, S. Yunoki, and E. Dagatto, Science 283, 2034 (1999).

${ }^{17}$ A. Moreo, M. Mayr, A. Feiguin, S. Yunoki, and E. Dagotto, Phys. Rev. Lett. 84, 5568 (2000).

${ }^{18}$ S. Yunoki, J. Hu, A. L. Malvezzi, A. Moreo, N. Furukawa, and E. Dagotto, Phys. Rev. Lett. 80, 845 (1998).

${ }^{19}$ M. Uehara, S. Mori, C. H. Chen, and S. W. Cheong, Nature (London) 399, 560 (1999).
${ }^{20}$ C. Martin, A. Maignan, M. Harvieu, and B. Raveau, Phys. Rev. B 60, 12191 (1999).

${ }^{21}$ C. martin, A. Maignan, F. Damay, and B. Raveau, Solid State Chem. 134, 198 (1997).

${ }^{22}$ D. D. Sarma, N. Shanthi, S. R. Krishnakumar, T. Saitoh, T. Mizokawa, A. Sekiyama, K. Kobayashi, A. Fujimori, E. Weschke, R. Meier, G. Kaindl, Y. Takeda, and M. Takano, Phys. Rev. B 53, 6873 (1996).

${ }^{23}$ T. Saitoh, A. E. Bocquet, T. Mizokawa, H. Namatame, A. Fujimori, M. Abbate, Y. Takeda, and M. Takano, Phys. Rev. B 51, 13942 (1995).

${ }^{24}$ K. Ebata, H. Wadati, M. Takizawa, A. Fujimori, A. Chikamatsu, H. Kumigashira, M. Oshima, Y. Tomioka, and Y. Tokura, Phys. Rev. B 74, 064419 (2006).

${ }^{25}$ M. K. Dalai, P. Pal, B. R. Sekhar, N. L. Saini, R. K. Singhal, K. B. Garg, B. Doyle, S. Nannarone, C. Martin, and F. Studer, Phys. Rev. B 74, 165119 (2006).

${ }^{26}$ M. K. Dalai, P. Pal, R. Kundu, B. R. Sekhar, S. Banik, A. K. Shukla, S. R. Barman, and C. Martin, Physica B 405, 186 (2010).

${ }^{27}$ P. Pal, M. K. Dalai, R. Kundu, M. Chakraborty, B. R. Sekhar, and C. Martin, Phys. Rev. B 76, 195120 (2007).

${ }^{28}$ P. Pal, M. K. Dalai, R. Kundu, B. R. Sekhar, and C. Martin, Phys. Rev. B 77, 184405 (2008).

${ }^{29}$ M. Merz, N. Nucker, E. Pellegrin, P. Schweiss, S. Schuppler, M. Kielwein, M. Knupfer, M. S. Golden, J. Fink, C. T. Chen, V. Chakarian, Y. U. Idzerda, and A. Erb, Phys. Rev. B 55, 9160 (1997).

${ }^{30}$ F. M. F. de Groot, M. Grioni, J. C. Fuggle, J. Ghijsen, G. A. Sawatzky, and H. Petersen, Phys. Rev. B 40, 5715 (1989).

${ }^{31}$ H. Kurata, E. Lefevre, C. Colliex, and R. Brydson, Phys. Rev. B 47, 13763 (1993). 
${ }^{32}$ M. Abbate, F. M. F. de Groot, J. C. Fuggle, A. Fujimori, O. Strebel, F. Lopez, M. Domke, G. Kaindl, G. A. Sawatzky, M. Takano, Y. Takeda, H. Eisaki, and S. Uchida, Phys. Rev. B 46, 4511 (1992).

${ }^{33}$ J.-H. Park, C. T. Chen, S. W. Cheong, W. Bao, G. Meigs, V. Chakarian, and Y. U. Idzerda, Phys. Rev. Lett. 76, 4215 (1996).

${ }^{34}$ O. Toulemonde, F. Millange, F. Studer, B. Raveau, J. H. Park, and C. T. Chen, J. Phys. Condens. Matter 11, 109 (1999).
${ }^{35}$ N. Mannella, A. Rosenhahn, M. Watanabe, B. Sell, A. Nambu, S. Ritchey, E. Arenholz, A. Young, Y. Tomioka, and C. S. Fadley, Phys. Rev. B 71, 125117 (2005).

${ }^{36}$ E. Pollert, S. Krupicka, and E. Kuzmicova, J. Phys. Chem. Solids 43, 1137 (1982).

${ }^{37}$ Z. Jirak, S. Krupicka, Z. Simsa, M. Dlouha, and S. Vratislav, J. Magn. Magn. Mater. 53, 153 (1985). 